\title{
Intrapersonal, social and physical environmental determinants of moderate-to-vigorous physical activity in working-age women: a systematic review protocol
}

Stephanie A Prince ${ }^{*}$, Jennifer L Reed ${ }^{1}$, Kara A Nerenberg ${ }^{2}$, Elizabeth A Kristjansson ${ }^{3}$, Swapnil Hiremath ${ }^{4}$, Kristi B Adamo ${ }^{5}$, Heather E Tulloch', Kerri-Anne Mullen', J George Fodor ${ }^{1}$, Erica Wright ${ }^{6}$ and Robert D Reid ${ }^{1}$

\begin{abstract}
Background: The majority of North American adult females do not meet current physical activity recommendations (150 min of moderate-to-vigorous intensity physical activity (MVPA) per week accrued in $\geq 10$ min bouts) ultimately placing themselves at increased risk of morbidity and mortality. Working-age females face particular challenges in meeting physical activity recommendations as they have multiple demands, including occupational, family and social demands. To develop effective interventions to increase MVPA among working-age females, it is necessary to identify and understand the strongest modifiable determinants influencing these behaviours. Therefore, the objective of this systematic review is to examine the available evidence to identify intrapersonal, social and environmental determinants of MVPA among working-age females.

Methods/Design: Six electronic databases will be searched to identify all prospective cohort studies that report on intrapersonal, social and/or environmental determinants of MVPA in working-age females. Grey literature sources including theses, published conference abstracts and websites from relevant organizations will also be included. Articles that report on intrapersonal (e.g. health status, self-efficacy, socio-economic status (SES), stress, depression), social environmental (e.g. crime, safety, area SES, social support, climate and capital, policies), and environmental (e.g. weather, workplace, home, neighbourhood, recreation environment, active transportation) determinants of MVPA in a working-age (mean age 18-65 years) female population will be included. Risk of bias will be assessed within and across all included studies using the Tool to Assess Risk of Bias in Cohort Studies and the Grades of Recommendation, Assessment, Development and Evaluation approach. Harvest plots will be used to synthesize results across all determinants, and meta-analyses will be conducted where possible among studies with sufficient homogeneity.
\end{abstract}

Discussion: This review will provide a comprehensive examination of evidence in this field and will serve to highlight gaps for future research on the determinants of MVPA in working-age females and ultimately inform intervention design.

Systematic review registration: PROSPERO: CRD42014009750.

Keywords: Moderate-to-vigorous physical activity, Socio-ecological determinants, Working females

\footnotetext{
* Correspondence: sprinceware@ottawaheart.ca

'Division of Prevention and Rehabilitation, University of Ottawa Heart

Institute, 40 Ruskin Street, Ottawa, ON K1Y 4 W7, Canada

Full list of author information is available at the end of the article
} 


\section{Background}

In North America, only $3 \%$ to $14 \%$ of adult females [1,2] achieve at least $150 \mathrm{~min}$ (in bouts of $10 \mathrm{~min}$ or more) of moderate-to-vigorous intensity physical activity (MVPA) per week as recommended by the World Health Organization [3]. Research consistently shows that greater amounts and higher intensities of MVPA are protective against weight gain, chronic diseases including hypertension, heart disease, type 2 diabetes, colon cancer, breast cancer, and osteoporosis, and premature mortality [4-6].

The majority of adults spend a large portion of their days at work, limiting the amount of free time available for engaging in leisure pursuits such as physical activity $[7,8]$. Free time refers to the time in a day that remains after work and other necessary daily activities that have been performed and provides an important opportunity for rest, social interactions, leisure pursuits, and selfreflection [9]. Most working-age females generally report having even less free time than males, making it difficult for them to achieve the recommended levels of MVPA [8-10]. These females have to overcome several factors that compete for their time on a daily basis including occupational, family and social demands [10,11]. Working females have consistently reported difficulty in engaging in MVPA due to a variety of these factors [11]. Interestingly, employed females are more likely than males to report work demands as a barrier to their physical activity [12]. Females with children are also less physically active than females without children, and those with young children ( $<6$ years) are less active than males with children the same age [13]. Females are also less active than their male peers $[14,15]$ and are more likely to become inactive if they get married, have children or transition to paid employment [16-18].

Research examining the determinants of adult (both male and female) physical activity has widely applied socio-ecology theory as a framework [19-22]. The socioecology theory recognizes that individual behaviours are likely dependent on the dynamic relationships between multiple determinants (i.e. biology, motivation, self-efficacy, socio-cultural, policy, built and natural environments) across several levels (i.e. intrapersonal, interpersonal, workplace, community) [23]. Sallis et al. proposed an ecological model of MVPA as a conceptual approach to understanding the determinants of time spent being physically active within different domains (i.e. recreation, transport, household, and occupation) (Figure 1) [24]. Their model provides a schematic framework that recognizes the possible behaviour settings and contextual factors which have the capacity to influence time spent being physically active in these specific domains.

To develop effective interventions and appropriate health policies, it is necessary to identify and understand the potentially modifiable factors that influence a female's propensity to be physically active and achieve the recommended $150 \mathrm{~min}$ of MVPA per week [3]. Previous reviews, though limited in number and now out-dated, examined the literature for factors related to physical activity uptake and adherence among females [25,26]. Importantly, none to date has specifically focused on working females who are likely to present with unique challenges in achieving work-life balance. Consequently, there is a need to identify which of these factors most strongly predict working-age females' total, as well as domain-specific (work, home, leisure, transport) MVPA.

In addition to the growing body of evidence around determinants of MVPA, numerous interventions have been designed and tested to increase MVPA specifically among females, though fewer among working-age females, specifically [27-30]. Given that female adults spend the majority of their day at work and report limited free time, workplaces represent important settings for physical activity interventions. Although interventions are being proposed and tested [31,32], there has been relatively little work examining and synthesizing the available evidence to identify the strongest determinants of domain specific MVPA and/or total time spent in MVPA among working-age females, which may represent the best targets for intervention in this population. It is, therefore, imperative that the most important modifiable factors of MVPA among working-age females be identified for the development of effective interventions and appropriate health policies. In response to this need, the objective of the proposed systematic review is to identify intrapersonal, social and environmental determinants of domain-specific (work, home, transport, leisure) and total time spent in MVPA among workingage females.

\section{Methods/design \\ Study design}

A systematic review and meta-analysis will be undertaken to identify common and important intrapersonal, social and environmental determinants of MVPA in working-age females in high-income countries. The systematic review will adhere to the reporting guidelines of the Preferred Reporting Items for Systematic Reviews and Meta-Analyses (PRISMA) statement [33] and will meet the items outlined in A Measurement Tool to Assess Systematic Reviews (AMSTAR) checklist [34,35].

\section{Study registration}

This systematic review has been prospectively registered with PROSPERO (registration number CRD42014009750; http://www.crd.york.ac.uk/PROSPERO). 


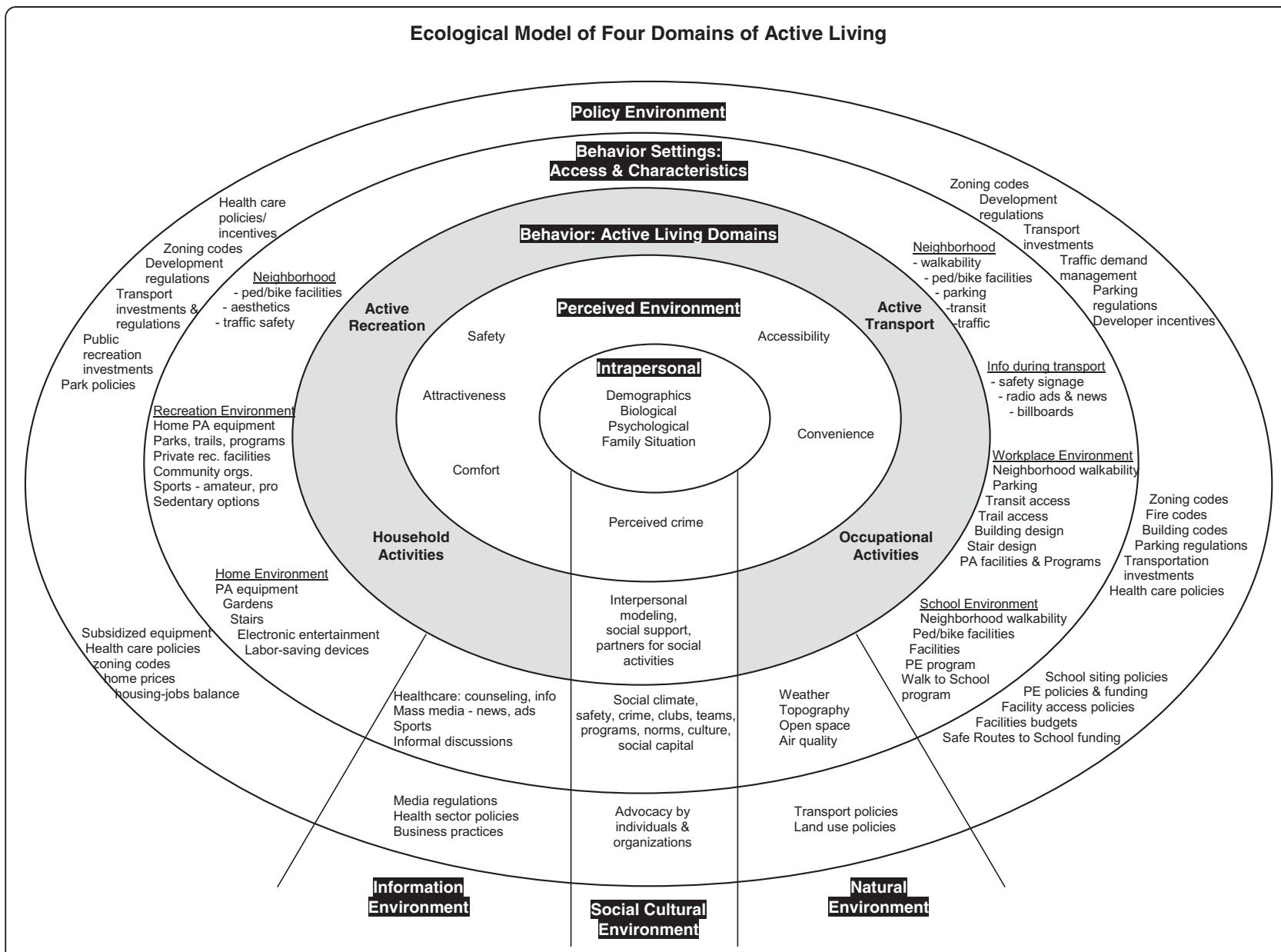

Figure 1 Ecologic model of the four domains of active living/MVPA [24] (reproduced with permission from the author and publisher).

\section{Criteria for considering studies for this review Types of participants}

Studies will be included if the sample is largely comprised of working-age females $(\geq 80 \%$ or where female data can be separated), with a mean age between 18 and 65 years. Further, only studies from high-income countries will be included (those with a gross national income (GNI) per capita of $\$ 12,746$ or more in 2013) [36]. Participant characteristics will be extracted for planned sub-group analyses (e.g. younger versus older, special populations such as types of occupation groups, work status (part-time, full-time, casual), women with children versus those without, and disease status). Studies with a mean age lower than 18 years or greater than 65 years or use of non-human participants (e.g. rats) will be excluded.

\section{Types of exposures}

Using an ecological model of active living [24], the review will examine intrapersonal (e.g. health status, selfefficacy, working status, socio-economic status, family status), social environmental (e.g. crime, safety, social support, social climate, social capital, policies), and physical environmental including natural environment and behaviour settings (e.g. weather, workplace environment, home environment, neighbourhood design, recreation environment, active transportation) determinants of MVPA among working-age females. The ecological model of active living recognizes that domain-specific physical activities are largely influenced by factors in the behaviour settings themselves, including the neighbourhood, recreation, home, workplace, school and transportation environments. Further, the socialcultural environment, policy environment, information environment and natural environments are recognized as playing a role within each of these domains and settings. The exposures will be identified as either objectively measured (e.g. crime rate in a neighbourhood) or perceived factors (e.g. feelings of safety in a neighbourhood). Studies where individual determinants cannot be isolated such as in the case of composite questionnaire scales will be excluded. 


\section{Types of comparators}

Comparator or control groups are not applicable in this research as only prospective cohort studies are eligible.

\section{Types of outcomes}

As the main outcome of interest is MVPA level, studies that do not report on this will be excluded. MVPA is defined as a behaviour with an energy expenditure $\geq 3$ metabolic equivalents (METs) $\left(1\right.$ METs $\left.=1\left(\frac{\mathrm{kcal}}{\mathrm{kg} * h}\right)\right), \geq 40 \%$ of $\mathrm{VO}_{2}$ reserve, $\geq 45 \%$ peak $\mathrm{VO}_{2}, \geq 55 \%$ of peak heart rate, $\geq 12$ on the Ratings of Perceived Exhaustion Scale (RPEs), or $>100$ steps per min [37-40]. For example, MVPA can be achieved by walking more than $3.2 \mathrm{~km} / \mathrm{h}$, cleaning (vacuuming, washing a car), or bicycling for pleasure [38]. Measures of association and risk between an exposure and MVPA will be captured from all studies. Depending on the number of studies identified for specific domains of MVPA, the review may be divided into multiple papers to better analyse determinants relevant to specific domains. Measures of time spent in total or domain-specific MVPA (e.g. min per day of MVPA from active recreation, transportation, household chores or occupation) or intensity (i.e. moderate and/or vigorous intensity physical activity) and where possible, a measure of variance around the outcome (e.g. standard error, 95\% confidence intervals) will be extracted from all included studies regardless of the unit of measurement or method of measurement. MVPA can be either objectively measured (e.g. indirect calorimetry, accelerometers, activity monitors, observed patterns) or self-reported (e.g. physical activity questionnaire, journal or $\log )$. Potential and known health sequelae of MVPA (e.g. obesity, diabetes) are not of interest.

\section{Type of studies}

To obtain results describing determinants of MVPA, the systematic review will include all published and unpublished prospective cohort studies that quantify the association between a risk factor/determinant and the level of MVPA in working-age females. Although no language restrictions will be imposed in the search, only articles published in English or French will be included. A summary of this evidence and the confidence in this evidence will be conducted using Cochrane's Grades of Recommendation, Assessment, Development and Evaluation (GRADE) approach [41] to increase internal validity of the review.

\section{Search methods for the identification of studies}

A sensitive and comprehensive search strategy has been designed in collaboration with a research librarian (EW) and includes a search of six electronic databases: Ovid MEDLINE(R) In-Process (1946 to present);
EBM Reviews-Cochrane Central Register of Controlled Trials (present); Embase Classic + (1947 to present); Ovid PsycINFO (1806 to present); SPORTDiscus (1830 to present); and dissertations and theses (1861 to present). The strategy is illustrated using the MEDLINE search as an example (Table 1) and will be modified according to the indexing systems of the other databases. Grey literature (non-peer reviewed works) that meets the inclusion criteria will be obtained including the following: published conference abstracts indexed under the bibliographic databases, published lists of theses and dissertations, government reports, and unpublished data and manuscripts (provided by original authors). Government reports will be searched using the Google search engine using a combination of key text words. The Google search engine will be used to identify studies that are published in non-indexed journals. Unpublished data and manuscripts will be solicited from authors of studies that report collecting MVPA, but in which, this data is not available within a published manuscript. Knowledgeable researchers in the field including those affiliated with the International Physical Activity and the Environment Network (www.ipenproject.org) will be solicited to identify other studies of interest. Finally, the bibliographies of all studies selected for the review will also be examined to identify further studies, as well as those of previous reviews.

\section{Selection of studies}

Citations retrieved from the search will be imported into EndNote X7 (Thompson Reuters, San Francisco, CA, USA), and duplicates will be removed using the 'duplicate' function. Remaining duplicates will be removed manually. Study eligibility assessment will be done independently in two stages by two reviewers (SAP, JLR). In the first stage, the reviewers will independently screen the titles and abstracts of all studies to identify eligible abstracts. In the second stage, the full texts of all abstracts that met the inclusion criteria or did not have insufficient information to judge eligibility in the abstract will be obtained and reviewed. If disagreements between the reviewers occur, consensus will be achieved through discussion and/or with the assistance of a third reviewer (EAK or RDR). Agreement will be measured at each stage. Reviewers will not be blinded to the authors or journals when screening articles.

\section{Data collection}

Prior to data extraction, a standardized data extraction form will be created and pilot tested by the research team using a subset of the included studies. The extraction form will be modified based on feedback from the extractors to improve its usability and ensure that complete and appropriate information is obtained. Standardized data 
Table 1 Sample MEDLINE search strategy

\begin{tabular}{cl}
\hline \multicolumn{3}{c}{ Search terms } \\
\hline Outcome terms \\
2 & Exp exercise/ \\
3 & (moderate adj2 vigorous).tw. \\
4 & MVPA.tw. \\
5 & Physical* activit*.tw. \\
6 & Exp sports/ \\
7 & Dancing/ \\
8 & Motor activity/ \\
9 & Physical fitness/ \\
10 & Aerobics.tw. \\
11 & ((moderate or vigorous or aerobic) adj2 exercise*).tw. \\
12 & ((moderate or vigorous or aerobic) adj2 activit*).tw. \\
13 & Running.tw. \\
14 & (cycling or biking).tw. \\
15 & Swimming.tw. \\
16 & Walking.tw. \\
17 & (physical* adj (fit* or train* or exercise*)).tw. \\
18 & "high intensit*" adj2 (exercise* or activit*).tw. \\
& or/1-17
\end{tabular}

Physical environment terms

\begin{tabular}{|c|c|}
\hline 19 & Environment design/ \\
\hline 20 & Residence characteristics/ \\
\hline 21 & Poverty areas/ \\
\hline 22 & Built environment*.tw. \\
\hline 23 & (walkable or walkability).tw. \\
\hline 24 & (active adj (travel* or transportation or commut*)).tw. \\
\hline 25 & $\begin{array}{l}\text { ((walking or pedestrian or cycling or bicycle or bike) adj } \\
\text { (trail }^{*} \text { or path* or route* or lane* or infrastructure)).tw. }\end{array}$ \\
\hline 26 & ((road or street) adj connectivity).tw. \\
\hline 27 & (community adj2 (feature* or characteristic*)).tw. \\
\hline 28 & Community design.tw. \\
\hline 29 & Neighbo?rhood*.tw. \\
\hline 30 & Sidewalk*.tw. \\
\hline 31 & Green space.tw. \\
\hline 32 & Parks.tw. \\
\hline 33 & Public facilities/ \\
\hline 34 & Fitness centers/ \\
\hline 35 & ((sport* or recreation* or exercise) adj facilit*).tw. \\
\hline 36 & ("land use" adj2 mix*).tw. \\
\hline 37 & (environment* adj (factor* or correlate* or determinant*)).tw. \\
\hline 38 & Weather/ \\
\hline 39 & Weather.tw. \\
\hline 40 & (gym or gyms).tw. \\
\hline 41 & $(($ fitness or recreation*) adj (centre* or center*)).tw. \\
\hline 42 & or/19-41 \\
\hline
\end{tabular}

Table 1 Sample MEDLINE search strategy (Continued)

Social environment terms

$\begin{array}{ll}43 & \text { Social environment/ } \\ 44 & \text { Community networks/ } \\ 45 & \text { Crime/ } \\ 46 & \text { ((safe* or unsafe) adj2 neighbo?rhood*).tw. } \\ 47 & \text { Social support/ } \\ 48 & \text { Exp socioeconomic factors/ } \\ 49 & \text { Culture/ } \\ 50 & \text { Cultural characteristics/ } \\ 51 & \text { (social* adj (capital or support* or influence* or environment* } \\ 52 & \text { or connect* or correlate* or factor } \text { (s).tw. }^{*} \\ 53 & \text { (socioeconomic or socio-economic).tw. } \\ 54 & \text { (sociodemographic }{ }^{*} \text { or socio-demographic*).tw. } \\ 55 & \text { (cultural adj (factor* or correlate* or influence*).tw. } \\ 56 & \text { Exp socioeconomic factors/ } \\ 57 & \text { Public policy/ } \\ 58 & \text { Health policy/ }\end{array}$

Intrapersonal terms

$\begin{array}{ll}59 & \text { Self efficacy/ } \\ 60 & \text { Motivation/ } \\ 61 & \text { Health status/ } \\ 62 & \text { Attitude to health/ } \\ 63 & \text { Health knowledge, attitudes, practice/ } \\ 64 & \text { Health behaviour/ } \\ 65 & \text { Self efficacy.tw. } \\ 66 & \text { Motivation.tw. } \\ 67 & \text { or/59-66 } \\ 68 & 42 \text { or } 58 \text { or } 67 \\ 69 & 18 \text { and } 68\end{array}$

Population and study design limits

$\begin{array}{ll}70 & \text { Child/not exp adult/ } \\ 71 & \text { Adolescent/not exp adult/ } \\ 72 & 70 \text { not } 71 \\ 73 & 73 \text { not } 72 \\ 74 & \text { Exp aged/not adult/ } \\ 75 & 74 \text { not } 75 \\ 76 & \text { Wom?n*.tw. } \\ 77 & \text { Exp women/ } \\ 78 & \text { Female*.tw. } \\ 79 & \text { Female/ } \\ 80 & \text { Womens health/ } \\ 81 & \text { or/77-81 } \\ 82 & 76 \text { and } 82 \\ 83 & \text { Exp animals/not humans/ } \\ 84 & 83 \text { not } 84\end{array}$




\section{Table 1 Sample MEDLINE search strategy (Continued)}

\begin{tabular}{ll}
\hline 85 & Epidemiologic studies/ \\
86 & Exp cohort studies/ \\
87 & (follow up adj (study or studies)).tw. \\
88 & (observational adj (study or studies)).tw. \\
89 & Longitudinal.tw. \\
90 & Cohort analy $\$ . t w$. \\
91 & (cohort adj (study or studies)).tw. \\
92 & Or/70-91 \\
93 & 69 and 92 \\
\hline
\end{tabular}

abstraction forms including quality assessments will be completed by one reviewer (SAP) and verified by another (JLR). If disagreements occur, consensus will be achieved through discussion and/or with assistant of a third reviewer (EAK or RDR). Reviewers will not be blinded to the authors or journals when extracting data.

From each included study, the following information will be extracted: lead author; year of publication; country of study; participant characteristics (age range and mean, sex distribution, health status, study setting); sample size; study design; length of follow-up (if applicable); exposure/determinant (separate entry for each determinant examined); measurement method for each exposure/ determinant (including whether it is self-reported or objectively measured); level of the determinant (e.g. individual, social environment, physical environment); whether MVPA is self-reported or objectively measured; whether MVPA is reported as a daily total or under a specific domain; measurement method and units of measurement of MVPA; analytical methods used (e.g. unadjusted, adjusted/multivariate regression); relationship between the exposure/determinant and MVPA (significant positive, negative or absence of association; and effect on MVPA (e.g. increase, no change, or decrease in MVPA). Authors of suspected duplicate reports (i.e. reports on the same population and relationships between determinant and MVPA) will be contacted and in cases where several publications report the same results from the same data source, only one study per data source/analysis will be retained to avoid double counting.

If a paper employs a measure that has the potential to capture MVPA (e.g. International Physical Activity Questionnaire, accelerometers, etc.) but does not report on these outcomes in the manuscript or if a paper describes a study protocol related to MVPA, the authors will be contacted to ascertain whether the MVPA results can be provided. A maximum of three e-mail attempts will be made to contact the lead author of these studies to obtain additional information.

\section{Risk of bias within studies}

Risk of bias will be assessed using the Tool to Assess Risk of Bias in Cohort Studies [42]. The tool includes an assessment of the following: selection bias (e.g. whether the exposed and unexposed groups were drawn from same population); performance bias (e.g. whether any co-interventions were present and if they influenced the exposed and unexposed similarly, confidence that the outcome of interest was not present at the start of the study); attrition bias (e.g. were the lengths of follow-up similar between exposed and unexposed); detection bias (e.g. confidence in the assessment of the exposures, outcomes and confounders, whether the study matched the exposed and unexposed groups for all variables associated with the outcome or was the analysis adjusted for these variables); and reporting bias (e.g. whether the outcomes were part of an a priori plan, whether the assessment of data could be reproduced). The risk of bias assessment will be carried out by two independent assessors (SAP and JLR), and if disagreements between assessors occur, consensus will be achieved through discussion with a third reviewer (RDR).

\section{Quality of the evidence}

The quality of the evidence for the relationship between each exposure and MVPA will be assessed as high, moderate, low or very low using Cochrane's GRADE approach [41]. Within this approach, non-randomized studies begin as low-quality evidence. In addition to study design, the quality of evidence will be rated upon possible risk of bias, imprecision, heterogeneity, indirectness, or suspicion of publication bias. Quality will be rated up if the summary of effect is large. GRADE suggests 'considering rating up quality of evidence one level when methodologically rigorous observational studies show at least a two-fold reduction or increase in risk, and rating up two levels for at least a five-fold reduction or increase in risk' [43]. GRADE Pro version 3.6.1 (GRADE Working Group) will be used to rate the quality of the evidence using the GRADE methodology.

\section{Planned analyses}

Summary tables will be created to describe the populations, interventions (if applicable) and outcomes of all studies. The relationships between a large number of exposures and different levels (i.e. individual, neighbourhood) with MVPA will be assessed using a variety of methods. Due to the variety in the exposures and metrics used in the studies, the review will use Harvest plots $[44,45]$ as a primary method of synthesis. Harvest plots allow results of the primary studies to be displayed across the various exposures and metric (e.g. perceived or objectively measured neighbourhood walkability) across various levels (i.e. individual, social environment, built 
environment) and across various outcome measurement methods (i.e. self-reported vs. objectively measured MVPA) to incorporate the strength of association, sample size, and study quality $[44,45]$. The Harvest plots will provide a graphical method to allow for a complete synthesis of the evidence and allow a comparison of the evidence across the various exposures.

Forest plots and meta-analyses will be created using Review Manager (RevMan) 5.3.3 (The Nordic Cochrane Centre, The Cochrane Collaboration, 2012) to synthesize the measures of effect (e.g. odds ratio, relative risk) and 95\% prediction intervals for each exposure on MVPA. A random effects meta-analysis will be used as populations and modifying factors are likely to be similar but not identical across all studies. Heterogeneity will be assessed using the $I^{2}$ statistic with values above $75 \%$ used to indicate high heterogeneity across studies [46]. Publication bias will be assessed using a funnel plot of the included studies' estimates of effect. The plots will be assessed both visually and by using Egger's test; with $p<0.10$ used to indicate the presence of a significant publication bias [47].

\section{Subgroup analyses}

In addition to the primary analyses proposed, several $a$ priori determined subgroup analyses will be performed when sufficient data are available. The analyses will examine differences between the following: age groups (e.g. 18-24 years versus 25-44 years versus 45-65 years); populations (e.g. healthy versus women with specific chronic diseases, between country groups i.e. North America versus Europe); occupation types (e.g. sedentary versus active); working status (i.e. full time, part time, homemaker, unemployed); self-reported and objectively measured exposures; self-reported and objectively measured MVPA; studies with high and low risk of bias and quality; different lengths of follow-up (e.g. <3 months, 3-6 months, $>6$ months); trends over time (e.g. by decade 1970s, 1980s, 1990s, 2000s, 2010s); and publication status (unpublished versus published results).

\section{Discussion}

This systematic review will be the first, to our knowledge, to critically examine and synthesize the available literature assessing the relationships between intrapersonal, social and environmental determinants of total and domain-specific MVPA among working-age females. The review will provide a comprehensive examination of the evidence in the field to date and will serve to highlight gaps where future research on the determinants of working-age female MVPA remains to be conducted. The use of the Harvest plots will allow researchers to visually examine all of the determinants across the multiple levels of influence that are related to MVPA including the strength and quality of the evidence. It is anticipated that this review will be useful for a variety of stakeholders including those looking to design interventions targeting the most important modifiable factors to increase MVPA among this high-risk female population.

Recommendations from previous reviews included that interventions should be multifaceted, targeting a combination of factors including psychosocial, social environmental, workplace setting, community environment and public policy, as well as be designed with recognition of the multiple roles of females and integrating physical activity into daily routines $[25,26]$.

\section{Abbreviations}

AMSTAR: a measurement tool to assess systematic reviews; GRADE: Grades of Recommendation, Assessment, Development and Evaluation;

METS: metabolic equivalents; MVPA: moderate-to-vigorous intensity physical activity; PRISMA: Preferred Reporting Items for Systematic Reviews and

Meta-analyses; RPE: rating of perceived exertion.

\section{Competing interests}

The authors declare that they have no competing interests.

\section{Authors' contributions}

SAP conceived the study, design and methodology, provided input for the bibliographic search strategy, and drafted and edited the manuscript. $J L R$ also conceived of the study, its design and coordination and provided critical revision of the manuscript. EW conceived the bibliographic search strategy, participated in its design and provided critical revision of the manuscript. JGF, SH, EAK, KAM, KAN, and HET participated in the design of the study, provided expert input, and critically reviewed the manuscript. RDR also conceived of the study, provided methodological input, and critically reviewed the manuscript. All authors read and approved the final manuscript.

\section{Acknowledgements}

The authors would like to thank Ms. Katelin Gresty for her review of the manuscript. SAP held a Gordon E. Allen Post-Doctoral Fellowship in Health Behaviours from the University of Ottawa Heart Institute (UOHI) and holds a Canadian Institutes of Health Research Fellowship and a Strategic Research Endowed Fellowship from the UOHI. JLR holds the Jan \& lan Craig Research Fellowship in Cardiovascular Prevention and Rehabilitation from the University of Ottawa Heart Institute.

\section{Author details}

${ }^{1}$ Division of Prevention and Rehabilitation, University of Ottawa Heart Institute, 40 Ruskin Street, Ottawa, ON K1Y 4 W7, Canada. ${ }^{2}$ Ottawa Hospital Research Institute, 725 Parkdale Avenue, Ottawa, ON K1Y 4E9, Canada. ${ }^{3}$ School of Psychology, University of Ottawa, 135 Jean Jacques Lussier, Ottawa, ON K1N 6 N5, Canada. ${ }^{4}$ Division of Nephrology, The Ottawa Hospital, 1053 Carling Avenue, Ottawa, ON K1Y 4E9, Canada. ${ }^{5}$ Healthy Active Living and Obesity Research Group, Children's Hospital of Eastern Ontario Research Institute, 401 Smyth Road, K1H 8 L1 Ottawa, ON, Canada. 'Berkman Library, University of Ottawa Heart Institute, 40 Ruskin Street, Ottawa, ON K1Y 4 W7, Canada.

Received: 17 September 2014 Accepted: 23 October 2014 Published: 4 November 2014

\section{References}

1. Colley RC, Garriguet D, Janssen I, Craig CL, Clarke J, Tremblay MS: Physical Activity of Canadian Adults: Accelerometer Results from the 2007 to 2009 Canadian Health Measures Survey. Statistics Canada: Ottawa; 2011.

2. Troiano RP, Berrigan D, Dodd KW, Mâsse LC, Tilert T, McDowell M: Physical activity in the United States measured by accelerometer. Med Sci Sports Exerc 2008, 40(1):181 
3. World Health Organization: Global Recommendations on Physical Activity for Health. Geneva, Switzerland: WHO Press; 2010.

4. Warburton D, Charlesworth S, Ivey A, Nettlefold L, Bredin S: A systematic review of the evidence for Canada's physical activity guidelines for adults. Int J Behav Nutr Phys Act 2010, 7(1):39.

5. Saris WH, Blair SN, Van Baak MA, Eaton SB, Davies PS, Di Pietro L, Fogelholm M, Rissanen A, Schoeller D, Swinburn B, Tremblay A, Westerterp KR, Wyatt H: How much physical activity is enough to prevent unhealthy weight gain? Outcome of the IASO 1st Stock Conference and consensus statement. Obes Rev 2003, 4(2):101-114.

6. Staiano AE, Reeder BA, Elliott S, Joffres MR, Pahwa P, Kirkland SA, Paradis G, Katzmarzyk PT: Physical activity level, waist circumference, and mortality. Appl Physiol Nutr Metab 2012, 37(5):1008-1013.

7. Canadian Fitness and Lifestyle Research Institute: How Canadians Spend their Time. Ottawa, Canada: Canadian Fitness and Lifestyle Research Institute; 2006.

8. United States Department of Labor: American Time Use Survey Summary 2014 June 26, 2014. In Available from: http://www.bls.gov/news.release/ atus.nro.htm

9. Chatzitheochari S, Arber S: Class, gender and time poverty: a time-use analysis of British workers' free time resources. Br J Sociol 2012, 63(3):451-471

10. Mattingly MJ, Blanchi SM: Gender differences in the quantity and quality of free time: the US experience. Soc Forces 2003, 81(3):999-1030.

11. Caperchoine C, Mummery WK, Joyner K: Addressing the challenges, barriers, and enablers to physical activity participation in priority women's groups. J Phys Act Health 2009, 6(5):589-596.

12. Cameron C, Craig CL, Stephens T, Ready TA: Increasing Physical Activity: Supporting an Active Workforce. Ottawa, Ontario: Canadian Fitness and Lifestyle Research Institute; 2002.

13. Adamo KB, Langlois KA, Brett KE, Colley RC: Young children and parental physical activity levels: findings from the Canadian health measures survey. Am J Prev Med 2012, 43(2):168-175.

14. Statistics Canada: Physical activity during leisure time, 2012-2013. In Available from: http://www.statcan.gc.ca/pub/82-625-x/2013001/article/ 11843-eng.htm.

15. Statistics Canada: Directly measured physical activity of Canadian adults, 2007 to 2011. In 2013. Available from: http://www.statcan.gc.ca/pub/82625-x/2013001/article/11807-eng.htm.

16. Brown WJ, Trost SG: Life transitions and changing physical activity patterns in young women. Am J Prev Med 2003, 25(2):140-143.

17. Dai S, Wang F, Morrison H: Predictors of decreased physical activity level over time among adults: a longitudinal study. Am J Prev Med 2014, 47(2):123-130.

18. Engberg $\mathrm{E}$, Alen M, Kukkonen-Harjula K, Peltonen JE, Tikkanen $\mathrm{HO}$, Pekkarinen $\mathrm{H}$ : Life events and change in leisure time physical activity: a systematic review. Sports Med 2012,42(5):433-447.

19. Prince SA, Kristjansson EA, Russell K, Billette JM, Sawada M, Ali A, Tremblay MS, Prud'homme D: A multilevel analysis of neighbourhood built and social environments and adult self-reported physical activity and body mass index in Ottawa, Canada. Int J Environ Res Public Health 2011, 8(10):3953-3978.

20. Sallis JF, Floyd MF, Rodríguez DA, Saelens BE: Role of built environments in physical activity, obesity, and cardiovascular disease. Circulation 2012, 125(5):729-737.

21. Elder JP, Lytle L, Sallis JF, Young DR, Steckler A, Simons-Morton D, Stone $E$, Jobe JB, Stevens J, Lohman T, Webber L, Pate R, Saksvig BI, Ribisl K: A description of the social-ecological framework used in the trial of activity for adolescent girls (TAAG). Health Educ Res 2007, 22(2):155-165.

22. Giles-Corti B, Donovan RJ: The relative influence of individual, social and physical environment determinants of physical activity. Soc Sci Med 2002, 54(12):1793-1812.

23. Sallis JF, Owen N, Fisher EB: Ecological models of health behavior. In Health Behavior and Health Education: Theory, Research, and Practice. 4th edition. Edited by Glanz K, Rimer BK, Viswanath KJ. San Francisco, California: Wiley \& Sons; 2008:465-486.

24. Sallis JF, Cervero RB, Ascher W, Henderson KA, Kraft MK, Kerr J: An ecological approach to creating active living communities. Annu Rev Public Health 2006, 27:297-322.

25. White JL, Ransdell LB, Vener J, Flohr JA: Factors related to physical activity adherence in women: review and suggestions for future research. Women Health 2005, 41(4):123-148.
26. Speck BJ, Harrell JS: Maintaining regular physical activity in women: evidence to date. J CardiovasC Nurs 2003, 18(4):282-291. quiz 292-283.

27. Bravata DM, Smith-Spangler C, Sundaram V, Gienger AL, Lin N, Lewis R, Stave CD, Olkin I, Sirard JR: Using pedometers to increase physical activity and improve health: a systematic review. JAMA 2007, 298(19):2296-2304.

28. Ogilvie D, Foster CE, Rothnie H, Cavill N, Hamilton V, Fitzsimons CF, Mutrie N, Scottish Physical Activity Research Collaboration: Interventions to promote walking: systematic review. BMJ 2007, 334(7605):1204.

29. Kahn EB, Ramsey LT, Brownson RC, Heath GW, Howze EH, Powell KE, Stone EJ, Rajab MW, Corso P: The effectiveness of interventions to increase physical activity: a systematic review. Am J Prev Med 2002, 22(4 Suppl):73-107.

30. Wilcox S, Parra-Medina D, Thompson-Robinson M, Will J: Nutrition and physical activity interventions to reduce cardiovascular disease risk in health care settings: a quantitative review with a focus on women. Nutr Rev 2001, 59(7):197-214

31. Ribeiro MA, Martins MA, Carvalho CR: Interventions to increase physical activity in middle-age women at the workplace: a randomized controlled trial. Med Sci Sports Exerc 2014, 46(5):1008-1015.

32. Tan AM, Lamontagne AD, Sarmugam R, Howard P: A cluster-randomised, controlled trial to assess the impact of a workplace osteoporosis prevention intervention on the dietary and physical activity behaviours of working women: study protocol. BMC Public Health 2013, 13:405.

33. Moher D, Liberati A, Tetzlaff J, Altman DG: Preferred reporting items for systematic reviews and meta-analyses: the PRISMA statement. BMJ 2009, 339:b2535.

34. Shea BJ, Grimshaw JM, Wells GA, Boers M, Andersson N, Hamel C, Porter AC, Tugwell P, Moher D, Bouter LM: Development of AMSTAR: a measurement tool to assess the methodological quality of systematic reviews. BMC Med Res Methodol 2007, 7:10

35. Shea BJ, Hamel C, Wells GA, Bouter LM, Kristjansson E, Grimshaw J, Henry DA, Boers M: AMSTAR is a reliable and valid measurement tool to assess the methodological quality of systematic reviews. J Clin Epidemiol 2009, 62(10):1013-1020.

36. The World Bank: Updated income classifications 2014 [July 21, 2014]. In Available from: http://data.worldbank.org/news/2015-country-classifications.

37. Mezzani A, Hamm LF, Jones AM, McBride PE, Moholdt T, Stone JA, Urhausen A, Williams MA, European Association for Cardiovascular Prevention and Rehabilitation; American Association of Cardiovascular and Pulmonary Rehabilitation; Canadian Association of Cardiac Rehabilitation: Aerobic exercise intensity assessment and prescription in cardiac rehabilitation: a joint position statement of the European Association for Cardiovascular Prevention and Rehabilitation, the American Association of Cardiovascular and Pulmonary Rehabilitation and the Canadian Association of Cardiac Rehabilitation. Eur J Prev Cardiol 2013 20(3):442-467

38. Ainsworth BE, Haskell WL, Whitt MC, Irwin ML, Swartz AM, Strath SJ, O'Brien WL, Bassett DR Jr, Schmitz KH, Emplaincourt PO, Jacobs DR Jr, Leon AS: Compendium of physical activities: an update of activity codes and MET intensities. Med Sci Sports Exerc 2000, 32(9 Suppl):S498-S504.

39. Marshall SJ, Levy SS, Tudor-Locke CE, Kolkhorst FW, Wooten KM, Ji M, Macera CA, Ainsworth BE: Translating physical activity recommendations into a pedometer-based step goal: 3000 steps in 30 minutes. Am J Prev Med 2009, 36(5):410-415

40. Tudor-Locke C, Sisson SB, Collova T, Lee SM, Swan PD: Pedometer-determined step count guidelines for classifying walking intensity in a young ostensibly healthy population. Can J App/ Physio/ 2005, 30(6):666-676.

41. Guyatt GH, Oxman AD, Vist GE, Kunz R, Falck-Ytter Y, Alonso-Coello P, Schünemann HJ, GRADE Working Group: GRADE: an emerging consensus on rating quality of evidence and strength of recommendations. BMJ 2008, 336(7650):924-926.

42. Busse JW, Guyatt GH: Tool to assess risk of bias in cohort studies. In 2014. Available from: http://distillercer.com/resources/.

43. Guyatt GH, Oxman AD, Sultan S, Glasziou P, Akl EA, Alonso-Coello P, Atkins D, Kunz R, Brozek J, Montori V, Jaeschke R, Rind D, Dahm P, Meerpohl J, Vist G, Berliner E, Norris S, Falck-Ytter Y, Murad MH, Schünemann HJ, GRADE Working Group: GRADE guidelines: 9: rating up the quality of evidence. J Clin Epidemiol 2011, 64(12):1311-1316

44. Crowther M, Avenell A, MacLennan G, Mowatt G: A further use for the harvest plot: a novel method for the presentation of data synthesis. Res Synthesis Methods 2011, 2(2):79-83. 
45. Ogilvie D, Fayter D, Petticrew M, Sowden A, Thomas S, Whitehead M, Worthy G: The harvest plot: a method for synthesising evidence about the differential effects of interventions. BMC Med Res Methodol 2008, 8:8.

46. Higgins JP, Thompson SG, Deeks JJ, Altman DG: Measuring inconsistency in meta-analyses. BMJ 2003, 327(7414):557-560.

47. Egger M, Davey Smith G, Schneider M, Minder C: Bias in meta-analysis detected by a simple, graphical test. BMJ 1997, 315(7109):629-634.

doi:10.1186/2046-4053-3-132

Cite this article as: Prince et al:: Intrapersonal, social and physical

environmental determinants of moderate-to-vigorous physical activity

in working-age women: a systematic review protocol. Systematic Reviews

2014 3:132.

\section{Submit your next manuscript to BioMed Central and take full advantage of:}

- Convenient online submission

- Thorough peer review

- No space constraints or color figure charges

- Immediate publication on acceptance

- Inclusion in PubMed, CAS, Scopus and Google Scholar

- Research which is freely available for redistribution 